\title{
Non-local mass ejection development with and without magnetic reconnections
}

\author{
I. S. Veselovsky ${ }^{1}$ \\ ${ }^{1}$ Skobel'tsyn Institute of Nuclear Physics, Moscow State University, Moscow, Russia email: \\ veselov@dec1.sinp.msu.ru
}

\begin{abstract}
Available YOHKON, TRACE, SOHO/EIT and CORONAS-F data are interpreted as evidences of the non-local coronal mass ejection initiation processes when rapidly increasing electric currents appear in the coronal loops and arcades. These electric currents are driven by subphotospheric processes. Self-similar plasma motions are due to the electric drifts in crossed inductive electric and magnetic fields. Expanding non-planar loops often mimic "reconnection" features, being in reality projection effects of the "twist and fold" processes on the image plane. Among such patterns one can find examples of spurious x-points, concave fronts, blobs, islands, etc. Coronal mass ejections often proceed without large scale topological changes of magnetic fields. They are accompanied by enhanced small-scale turbulence.
\end{abstract}

\section{Introduction}

The dynamics of eruptive loop prominences and coronal mass ejections as observed by SOHO, TRACE and CORONAS-F show sometimes the complicated geometry and temperature distributions in the shape of twisted double or multiple spirals with hot and cold threads interweaved and expanding as a whole entity without visible topological changes during the initiation phase. The connectivity and the helicity were conserved during these events. The topological transition is not a necessary condition for the coronal mass initiation as in the case of the March 2, 2002 eruption and other examples. Nevertheless, some coronal mass ejections show topological transformations during the initiation of the eruption with the appearance of many small-scale dynamical elements. Hence, coronal mass ejections could proceed as laminar or turbulent processes with/without topological changes in one or several areas on the Sun. The important role in the development of the coronal mass ejections belongs to the Ampere forces and the Joule dissipation, but the topological transitions in the large scales are not necessary nor sufficient conditions for the eruption. Theoretical models are based on observations and include non-local connections between the magnetic field structures via the electric currents at different levels and places of the solar atmosphere.

\section{Spurious reconnections}

Sigmoids are sometimes assumed to be a manifestation of the s-shaped magnetic field lines in the solar atmosphere (Gibson et al. (1999)) . In reality, many of them are projection effects of two different field lines with the middle part rooted beneath the atmosphere (Zhukov et al. (2003)). Projection catastrophes (caustics) which are non-physical, but purely geometrical, could be misinterpreted as topological transitions or reconnections. It is especially true for several past observations of the chromospheric and coronal structures with a poor space - time and spectral resolution. Different morphological structures seen in the solar chromosphere and the corona on the limb and on the disk (loops, arcades, bushes, fans, rays, jets etc.) are formed by plasma inhomogeneities which are associated with self-consistent electric and magnetic fields on the Sun.

Many CMEs and eruptive prominences develop as expanding loops. Their rapid growth in size can be easily explained by the $E$ cross $B$ drift motion in the situation of the 'new magnetic flux' development, i.e. when $\frac{\partial B}{\partial t}$ is strong and positive. In this case, the 
characteristic velocity of the expansion is equal to $V \sim L / t$, where $L$ is the size of the loop, $t$ is the time scale of the magnetic field variation. Upper and larger loops expand faster. If we take $L \sim 100 \mathrm{Mm}, t \sim 20 \mathrm{~min}$, we obtain $V \sim 100 \mathrm{~km} / \mathrm{s}$. Topological changes are not necessary for this process. Recent observations of up-and-down motion in the solar corona were interpreted as an evidence of the magnetic reconnection (Simnett 2004). This interpretation is quite arbitrary, because the formation of new zero points in the magnetic field was not demonstrated. Topological changes are not necessary for such kind of motion, along and across the magnetic field (Simnett (2004)). We demonstrate this with several observational examples taken from recent SOHO and TRACE movies. Movies of helical structures, flux ropes, twisted loops, when projected on the image plane, show both outward and inward propagating features, which could be misinterpreted as an evidence of the large-scale magnetic reconnection (Plunkett et al. (2000)).

Helmet-like features with a sharply defined cusp have been seen in active regions in X-rays. They are similar to the optical helmet streamers, but with much smaller spatial scales. They could be long lasting during many hours or rapidly evolving during tens of minutes. (Cheng 1994). Loops are usually convex. If they are twisted, their projections on the picture plain could have self-crossing features, false x-points and concave profiles above them, which mimic "reconnection", "island", "bubbles", "detachment", "plasmoid", "concave slow shock fronts" etc. Deconvolution problems are not easy with the one - point vantage, but they could be solved with the future stereo images or even with high cadence movies, when secondary features suggest the global shape and its evolution. The attentive analysis of the simultaneous Yohkoh and SOHO/EIT movies brings many examples of this kind. We refer here "the best reconnection example" March 17-20, 1999 which is in reality the projection of this kind (http : //lasco-www.nrl.navy.mil/dailympg/1999_03). The cross-section of most loops seems to be constant, implying the presence of electric currents in the corona. Active-region loops are in the permanent motion, moving slowly outward, carrying plasma with them. During flares, loops often produce localized brightening at the base and later at the apex of the loop. The motions are faster at that time. A multi-wavelength approach could bring needed information about the overall geometry evolution. The magnetic field along such loops should be roughly constant, i.e. nearly equal in the legs and in the summits. This would mean $\beta>>1$ at the higher altitudes, if values of the order of tens-hundreds of Gauss are supposed. Sheared and twisted loops are also indicative of electric currents. Sheared loops are often stable for hours to days.

CMEs are much more common than cusped loops. They are rarely bright at the apex of the helmet, implying that reconnection is not going there. Expanding loops in active regions are not necessarily explained in terms of reconnection (Strong 1994). An interesting observation (A.Pevtsov, this conference) consists in the fact that several loops seems to have even thicker legs as compared with their summits. The suggested interpretation invokes fan-like structures with a vertical spine and a horizontal separator. Current sheets at the separating surfaces are quite possible. The increase of brightness of individual elements with an enhanced heating function (Joule dissipation or viscous friction) makes spurious impressions of "topological changes" in the magnetic field.

Thin loops are tracing magnetic field lines in the solar atmosphere. It is because of suppression of the plasma and heat diffusion across the field. The problem of the deconvolution is not trivial one because of projection effects on the plane of the image. Future stereoscopic observations are needed to remove some of existing ambiguities and uncertainties in the interpretation. The same transparent $3 D$ object seen from different vantage - points looks differently. The only exception is the sphere, which is projected on the plane as a circle. The circle seen at the image plane could be the projection, 
not only of the sphere, but infinite number of figures. The inverse problem of the $3 D$ reconstructions from projections on the plane is not easy and often ill-posed. The caution is needed every time in the interpretation which should be not biased by unjustified a priori model selection. The correct answer is possible only for well determined or overdetermined problems, when necessary and sufficient information is available for the unique solution. It is not the case in many instances in the solar physics.

\section{What causes the CME onset?}

There is no definite answer as yet. It is because of the poor knowledge of the physical parameters. Several ideas where suggested and discussed in the literature (see Forbes 2000). It was assumed that the hot plasma appears during solar flares and drives the expansion by an excessive gas pressure gradient. It is not always the case, because the cool plasma of prominences can be erupted even without strong flares and heating. Prominences, filaments or coronal channels (flux ropes) are ejected first (Plunkett et al. (1997)). Magnetic fields and electric currents are involved according to the general belief, but details remain to be clarified. According to one idea, the magnetic bubble or the cavity is formed around enhanced electric currents in the flux ropes with low beta. The whole system is than expelled by the Archimedean force. Another scenario considers Ampere forces acting on the electric currents in external magnetic fields. This scenario does not exclude previous ones and can also explain the situations with expelled CMEs propagating outwards as well as with submerged parts of the flux ropes which are propagating inwards or remaining intact. The enhancement of electric currents is a necessary ingredient in both these scenario. The key physical quantity - the electric current distribution is not well known during CMEs. The possible coupling of these electric currents to subphotospheric levels makes the CME initiation problem strongly non-local, contrary to assumptions of several theories considering a loss of a local stability of equilibrium of coronal magnetic fields.

The "storage models" consider the magnetic energy accumulation in the solar corona during hours and days before the CME. The instability can be triggered or spontaneous and could lead to a faster "energy release". The opposite view exists that the CME initiation process is directly driven from below by the electric currents and magnetic drifts of the plasma in the variable inductive crossed electric and magnetic fields. Real situations correspond to intermediate case between these two extremities, being closer to the first or to the second from case to case. Coronal mass ejection initiation processes are still not completely investigated both observationally and theoretically. The interplay between different space-time scales on the Sun is not quite clear in many instances. As a consequence, the free energy transformations and cause-sequence relations between observed structures and processes remain as in the case of the flare - CME dilemma. In reality, there is no such dilemma: both phenomena are two concurrent manifestations of one driving free energy source situated beneath the photosphere. Several connecting mechanisms are known to be important in providing this free energy to the solar atmosphere. They include plasma motions, heat conduction, electric currents, radiation and waves. Many details are not known and speculative.

Developments of the models for flare loops (see e.g. Fig. 37 in the paper Priest \& Forbes (2001)) sometimes neglect the existence of the vertical motions of the plasma of the loop summits as well as electric currents, plasma drift motions and Ampere forces here due to $E$ cross $B$ and $j$ cross $B$ actions (Eq.23 in the mentioned paper). Such preassumptions are too restrictive. An interesting observation belongs to J.-P. Delabondiniere (private communication, 2004), based on the investigation of the SOHO/EIT images. Eruptive prominences of filaments show many small-scale and dynamical features during "activation" process in the initial phase of the eruption. These features resemble many curls or 
chaotic splittings for numerous turns of the field lines tracing the overall shape before the eruption. When the eruption in the progress, these small scale features disappear. The impression is that turbulent small-scale features and the destabilization appear simultaneously. See e.g. the double cool and hot twisted loops eruption in the images of $\mathrm{SOHO} / \mathrm{EIT}$ in 19,5nm on March 2, 2002 around 12-15UT. The loop structure of the subsequent CME is seen in the LASCO C2/C3 images between 15:06 and 21:30. The $H \alpha-$ prominence reappeared at the same place a day later.

Complicated dynamical geometry of arcades is nicely seen in TRACE movies. Spurious reconnections and $\mathrm{X}$-points are recognized in projections of twisted loops. The same is true for "crossings of the loops". Brighter summits of the loops often look as detached elements or "plasmoids". Some of fuzzy and not well resolved (integrated over a broad spectral range) YOHKOH image are sometimes wrongly interpreted as manifestations of topological changes in the large scale magnetic field being in reality projection effects. For example, see http : //www.lmsal.co//Y POP/Nuggets/2000/001103.html and references there. The important role of projection effects needs more investigations (Vedenov et al. (2000)).

\section{Plasma drifts}

The role of plasma drifts in the eruptive processes on the Sun was considered in the papers Veselovsky (1998), Veselovsky (2002). It is important to stress here, that preassumption that plasma "can not cross magnetic field lines" and "can not flow across magnetic field" are not invariant against the reference frame selection and not universal at least because of this reason. Electric fields should be treated carefully to avoid nonphysical interpretation of the plasma motions, even if they are locally frozen in magnetic fields. Globally it is not the case in practically interesting situations, which are common in the solar physics.

\section{Conclusions}

Eruptions in the shape of expanding loops often proceed without any large-scale topological changes of magnetic fields. Topological changes in the magnetic field are not necessary for eruptions but they could happen during CMEs and eruptive flares, but they Nevertheless, the processes are complicated and have many space-time scales. Small-scale and rapid transformations demonstrate numerous "reconnections", i.e. the appearance of new zero points in the magnetic field.

\section{Acknowledgements}

The work is partially supported by the INTAS grant 03-51-6206, RFBR grant 04-0216736, Programs "University of Russia" and "Astronomia".

\section{References}

Gibson, S. E. et al. 1999 Proc. 8th SOHO Workshop. ESA SP-466, 331-334.

Zhukov, A. N. et al. 2003 In Solar Wind Ten.AIP CP679, pp. 711-717. Melville, N. Y.

Simnett, G. M. 2004 A\&A 416, 759-764.

Plunkett, S. P. et al. 2000 Solar Phys. 194(2), 371-391.

Cheng, C-C. 1994 Space Sci.Rev 70, 273-278.

Strong, K. T. and the Yohkoh Team. 1994 Space Sci.Rev. 70, 133-142.

Forbes, T. G. 2000 J. Geophys.Res. 105, 23153-23165.

Plunkett, S. P. et al. 1997 Solar Phys. A 175 (2), 699-715.

Priest, E. R. \& Forbes, T. G. 2001 The Astron.Astrophys. Rev. DOI 10.1007/S001590100013

Veselovsky, I. S. 1998 In New Perspectives on Solar Prominences (Ed by D.Webb, D.Rust $\mathcal{E}$ B. Schmieder) ASP Conf.Ser., vol. 150, pp. 123-126. Astron.Soc.of Pacific.

Veselovsky, I. S. 2002 In SOLMAG: Magnetic Coupling of the Solar Atmosphere. ESA SP-505, pp. 603-606.

Vedenov, A. A. et al. 2000 Astron. Rep. 44(2), 112-121. 